This item was submitted to Loughborough's Research Repository by the author.

Items in Figshare are protected by copyright, with all rights reserved, unless otherwise indicated.

\title{
Design of a networked control system with random transmission delay and uncertain process parameters
}

PLEASE CITE THE PUBLISHED VERSION

PUBLISHER

(c) Taylor \& Francis

VERSION

AM (Accepted Manuscript)

LICENCE

CC BY-NC-ND 4.0

\section{REPOSITORY RECORD}

Yang, Ye, Yongji Wang, and Shuang-Hua Yang. 2019. "Design of a Networked Control System with Random Transmission Delay and Uncertain Process Parameters". figshare. https://hdl.handle.net/2134/4060. 
This item was submitted to Loughborough's Institutional Repository (https://dspace.lboro.ac.uk/) by the author and is made available under the following Creative Commons Licence conditions.

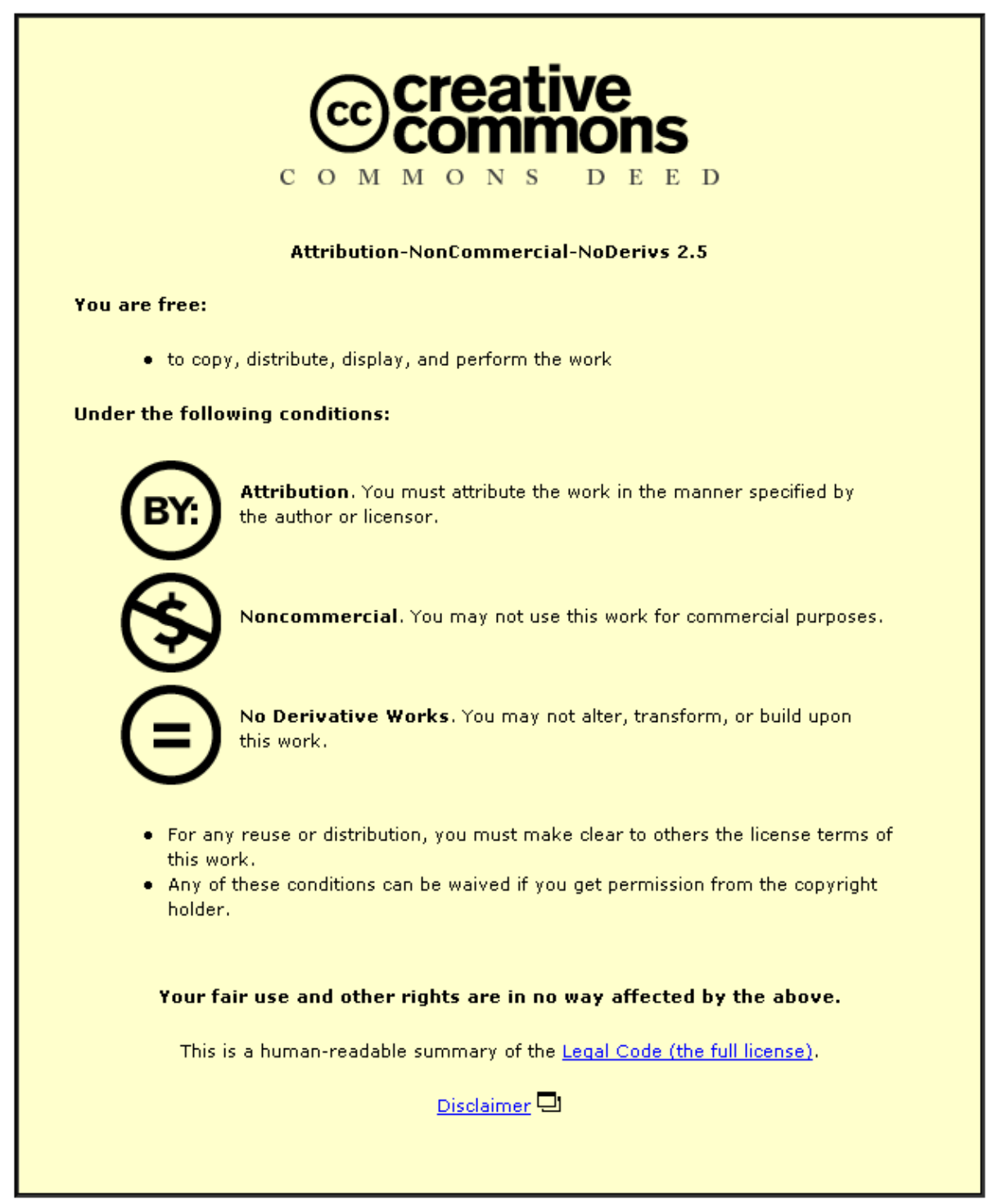

For the full text of this licence, please go to: http://creativecommons.org/licenses/by-nc-nd/2.5/ 


\title{
DESIGN OF A NETWORKED CONTROL SYSTEM WITH RANDOM TRANSMISSION DELAY AND UNCERTAIN PROCESS PARAMETERS
}

\author{
Ye Yang ${ }^{1}$, Yongji Wang ${ }^{1}$, and Shuang-Hua Yang ${ }^{2 *}$ \\ ${ }^{I}$ Department of Control Science and Engineering, Huazhong University of Science and Technology, \\ Wuhan, China \\ ${ }^{2}$ Department of Computer Science, Loughborough University, Loughborough, LE11 3TU, UK
}

\begin{abstract}
:
This paper discusses the compensation of the transmission delay in a networked control system (NCS) with a state feedback, which possesses a randomly varying transmission delay and uncertain process parameters. The compensation is implemented by using a buffer in the actuator node and a state estimator in the controller node. A Linear Matrix Inequality (LMI) based sufficient condition for the stability of the NCS under the designed compensation is proposed. The simulation results illustrate the efficiency of the compensation method.
\end{abstract}

Keywords: delay compensation; networked control system; time delay; stability analysis; uncertain linear system.

\footnotetext{
Corresponding author: Prof. S H Yang, in Computer Science at Loughborough University, UK. Email: s.h.yang@lboro.ac.uk
} 


\section{INTRODUCTION}

As the development of network technologies, more and more communication networks are used in industrial control. Applications of NCS include internet-based process control [1, 2, 3], internet-based robotics [4], fieldbus based NCS and Ethernet based NCS [5] etc. The advantages of NCS are reducing cost of cabling, ease system diagnosis and maintenance, increasing modularity and flexibility in system design.

However, the network transmission delays degrade the system dynamic performance and affect the stability of NCS. The network transmission delay is time varying and stochastic. There are two ways to overcome the transmission delay [2]. One is to improve the quality of network transmission by optimising communication protocols and adopting hardware devices with high performance so that the network-induced delays can be ignored. The other is to counteract the effect of network-induced delays on the system by using control theory approaches such as time-delay compensation, stochastic optimal control, predictive control, and robust control etc.

Rich literatures have been published on the NCSs. Zhang et al. [6] analysed the stability of NCSs, and achieved some important results based on the assumption that transmission delay is less than a sampling period and the data are transmitted in a single packet. Walsh et al. [7] considered a NCS in which the network is inserted between continuous plant and continuous controller, and introduced the notion of Maximal Allowable Transfer Interval (MATI), which is the maximum time interval between transfers of data from sensors to a controller. Their goal is to find the MATI that guarantees the stability of NCSs. Montestruque and Antsaklis [8] focused on reducing the network usage by using the knowledge of the plant dynamics. Necessary and sufficient conditions for stability of NCSs with a state feedback and an output feedback were derived respectively. Luck and Ray [9] modelled the network-induced delays as a constant by building buffers in the controller node and the actuator node respectively. The disadvantage of this method is prolonging the network-induced delay. Model Predictive Control (MPC) has been used in NCS to deal with random delay [2, 10, 11]. Gang et al. [10] considered MPC based NCS with stochastic time delay. Srinivasagupta et al. [11] used the global time stamps to determine the current and previous delays in NCS. Yang et al. [2] implemented a Dynamic Matrix Control (DMC) based time delay compensator over the Internet in a real laboratory rig. Liu et al. [12] introduced a hardware implementation for their MPC based NCS. Random communication delays, varying sensor delays and missing measurements have been also considered in the NCS research [13, 14]. 
Distinguish from the existing works this paper considers both stochastic transmission time delays in feedback and feed-forward channels and uncertain process parameters in NCS. A buffer is setup in the actuator node for saving the latest available corresponding control actions. The current time delay is calculated by comparing the current time with the time stamp received from the sensor node. If no time delay occurs in feedback channel, updated control actions for multi-step forward instants are generated in terms of the prediction of the states in the future instants. Otherwise, the control actions are generated based on the available measured states received from the sensor node for delayed instants. In both cases the control actions are generated in the control node and sent to the buffer in the actuator node. The actuator node applies only the control action for the current instant saved in the buffer to the plant. A sufficient stability condition is achieved for this particular model predictive control system with time delay compensation. These results may be useful in many practical situations.

This paper is organized as follows. In Section 2, a model of NCSs is given with a few of assumptions. A transmission delay compensation method is proposed in Section 3. The stability analysis for NCSs is addressed in Section 4. Section 5 illustrates the simulation results, which demonstrate the accuracy of the proposed method. Section 6 is the conclusions. The appendix gives the detailed proof of the stability theorem.

\section{MODELLING OF THE NCS}

Consider a class of ordinary NCS as shown in Fig. 1. It consists of a plant described in an uncertain discrete linear model.

$$
\left\{\begin{array}{l}
X(k+1)=(A+\Delta A) X(k)+(B+\Delta B) U(k) \\
Y(k)=C X(k)
\end{array}\right.
$$

and a discrete controller

$$
U(k)=h\left(k-r_{k}^{c a}\right)-K X\left(k-r_{k}\right), \quad k=0, \quad 1, \quad 2, \cdots,
$$

where $X \in R^{n_{1}}$ is the state vector; $U \in R^{n_{2}}$ is the control input vector; $A \in R^{n_{1} \times n_{1}}$ and $B \in R^{n_{1} \times n_{2}}$ are known constant real matrices; $\Delta A$ and $\Delta B$ are matrix-valued functions of appropriate dimension representing timevarying parameter uncertainties in the plant model. $K \in R^{n_{2} \times n_{1}}$ is the feedback control matrix. $r_{k}$ is the total transmission delay at instant $\mathrm{k}$ which equals the sum of the sensor-to-controller delay, denoted as $r_{k}^{s c}$, and the controller-to-actuator delay, denoted as $r_{k}^{c a}$. All the time delays $r_{k}, r_{k}^{s c}$ and $r_{k}^{c a}$ are rounded to an integer multiple of the sampling interval by including the various waiting times at the controller node and the actuator node into them respectively. The set-point $h(k)$ is normally set as zero without losing generalities. 
As usual, the parameter uncertainties considered are assumed to be norm bounded and satisfy

$$
\left[\begin{array}{ll}
\Delta A & \Delta B
\end{array}\right]=D F(k)\left[\begin{array}{ll}
E_{1} & E_{2}
\end{array}\right]
$$

where $D, E_{1}, E_{2}$ are known real matrixes of appropriate dimension that represent the structure of uncertainties, and $F(k) \in R^{S_{1} \times s_{2}}$ is unknown matrix function and satisfies

$$
F^{T}(k) F(k) \leq I
$$

in which $I$ is the identity matrix with an appropriate dimension.

The following assumption is made for the NCS: The total transmission delay $r_{k}$ is bounded and stochastically varying, i.e. $0 \leq r_{k} \leq m$, where $\mathrm{m}$ is an integer, which can be estimated according to the maximum time delay under the normal network condition. In case the network temperately collapses, the time delay will be greater than $m$, and the latest available control action will keep being used until the network recoveries.

Based on the above assumptions the actuators may receive zero, one, or more than one (up to $\mathrm{m}$ ) control action packets from the controller during a single sampling interval. If the actuators receive no control action packets during any sampling interval $\left[t_{k}, t_{k+1}\right), u_{k}$ in the last received control action packet will continue to act on the plant during the next sampling interval $\left[t_{k+1}, t_{k+2}\right)$. If the actuators receive more than one control action packets during any sampling interval $\left[t_{k}, t_{k+1}\right)$, only the most recent received control action packet is kept and the actuators will discard the others.

Concerning the random transmission time delay, the state feedback controller shown in Equation (2) can be represented as follows:

$$
\begin{gathered}
U(k)=-K \sum_{i=0}^{m} \delta\left(r_{k}-i\right) X(k-i), \quad k=0, \quad 1, \quad \cdots, \\
\delta\left(r_{k}-i\right)= \begin{cases}0 & r_{k} \neq i \\
1 & r_{k}=i\end{cases} \\
\sum_{i=0}^{m} \delta\left(r_{k}-i\right)=1
\end{gathered}
$$

$r_{k}$ represents the NCS random network transmission delay rounded to an integer, $r_{k} \in \wp=\left\{\begin{array}{ll}0, & 1, \cdots, \\ \end{array}\right\}$.

In spirit being similar to ones made by Nilsson [15], but easier to implement time delay compensation, a further rational assumption is made as follows: 
Let $\left\{r_{k}, k>0\right\}$ be a Markov chain with the state space $\wp=\{0,1, \cdots, m\}$ and the transition probabilities

are

$$
P^{r}\left\{r_{k+1}=j \mid r_{k}=i\right\}=P_{i j}^{r}, \forall i, j \in[0, \quad 1, \quad \cdots \quad m]
$$

Here $P_{i j}^{r} \geq 0$ and $\sum_{j=0}^{m} P_{i j}^{r}=1$.

\section{COMPENSATION OF TRANSMISSION DELAY AT FEEDBACK AND FEEDFORWARD CHANNELS}

For the sake of simplicity, a two-step design strategy is used in this study. A state feedback controller is firstly designed without considering the effect of NCS, and then a controller node and an actuator node are designed to compensate the effect of the sensor-to-controller delay and the controller-to-actuator delay. Fig. 2 illustrates the principle of the compensation of the transmission delay for NCS. A process model is located in the controller node in order to predict the future performance of the plant based on the latest available measured states. A buffer is located in the actuator node in order to compensate the effect of the transmission time delay.

\subsection{Compensation of the transmission delay at the sensor-to-controller channel}

Suppose the latest plant state received by the controller node is $X(k)$. The controller will predict the next $m$ plant states based on this measured plant state $X(k): \hat{X}(k+1), \hat{X}(k+2)$, to $\hat{X}(k+m)$, calculate the current and future $m$ control actions: $U(k), U(k+1), \cdots U(k+m)$ and then transmit them to the actuator node together with the time stamp received from the sensor node. The current control action $U(k)$, the prediction of the plant states and the future control actions at instants $k+1, k+2, \cdots k+m$ based on the measured plant state $X(k)$ are as follows:

$$
\left\{\begin{array}{l}
U(k)=-K X(k) \\
\hat{X}_{k+i \mid k}=(A-B K)^{i} X(k) \\
U_{k+i \mid k}=-K \hat{X}_{k+i \mid k} \\
i \in\left[\begin{array}{ll}
1, & 2, \cdots, \quad m]
\end{array}\right.
\end{array}\right.
$$

where $\hat{X}_{k+i \mid k}$ and $U_{k+i \mid k}$ are denoted as the prediction of the plant state and the future control action at instant $k+i$ based on the measured state $X(k)$. 
In general, if the latest available plant state received by the controller node is $X(k-j), j \in[0,1, \cdots, m]$, the prediction of the plant states and the future control actions based on them at instant $k+i$, $i \in[1, \quad 2, \quad \cdots, \quad m]$ can be represented as follows:

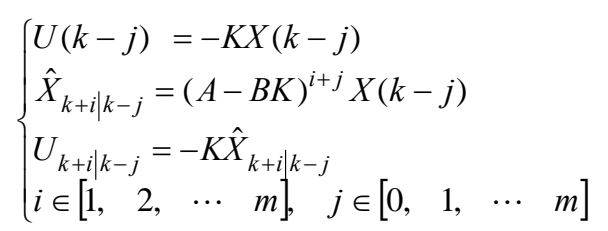

where $\hat{X}_{k+i \mid k-j}$ and $U_{k+i \mid k-j}$ are denoted as the prediction of the plant state and the future control action at instant $k+i$ based on the measured state $X(k-j)$.

\subsection{Compensation of the transmission delay at the controller-to-actuator channel}

Once a new measured plant state $X(k-j), \quad j \in[0,1, \cdots, m]$ is received by the controller node, the sensorto-controller time delay $r_{k}^{s c}$ will be calculated by comparing the current time with the time stamp received from the sensor node, and then rounded to an integer multiple of the sampling interval by adding a waiting time at the controller node into the delay, i.e. $j=r_{k}^{s c}$. Based on the latest available plant state $X\left(k-r_{k}^{s c}\right)$ the control actions $U(k), \quad U(k+1), \quad \cdots, \quad U(k+m)$ are calculated according to Equation (8) and then sent to the actuator node. Once the control action packet is received by the actuator node the controller-to-actuator time delay $r_{k}^{c a}$ will be calculated by comparing the current time with the time stamp received from the controller node and then rounded to an integer multiple of the sampling interval by adding a waiting time at the actuator node into the delay. The control actions available for the plant are $U\left(k-r_{k}^{c a}\right), \quad U\left(k+1-r_{k}^{c a}\right), \quad \cdots, \quad U\left(k+m-r_{k}^{c a}\right)$, which are saved in the buffer at the actuator node. The actuator node will choose $U(k)$ from the above list as the current control action acting on the plant. At instant $k+1$, i.e. the next sampling instant, if no any new control action packet is received from the controller node, $U(k+1)$ found from the control action packet $U\left(k-r_{k}^{c a}\right), \quad U\left(k+1-r_{k}^{c a}\right), \quad \cdots, \quad U\left(k+m-r_{k}^{c a}\right)$ will be used for the plant. If more than one control action packets are received only the packet with the latest time stamp will be saved in the buffer. This is used to deal with the situations of package disorder and package loss.

\subsection{Unified form of the state feedback controller}

Being similar with the uncompensated control action shown in Equation (5) the compensated control action obtained in the controller node and saved in the actuator node at instant $k$ can be formulised as follows: 


$$
\begin{aligned}
& U(k)=\sum_{i=0}^{m} \delta\left(r_{k}-i\right) U_{k \mid k-i} \\
& =-K \sum_{i=0}^{m} \delta\left(r_{k}-i\right) \hat{X}_{k \mid k-i}
\end{aligned}
$$

where $k=0, \quad 1, \cdots, \quad \delta\left(r_{k}-i\right)=\left\{\begin{array}{ll}0 & r_{k} \neq i \\ 1 & r_{k}=i\end{array}\right.$, and $\sum_{i=0}^{m} \delta\left(r_{k}-i\right)=1$

If the network temperately collapses $r_{k}$ may be greater than $m$. The latest available $U\left(k+m-r_{k}^{c a}\right)$ from the buffer will keep being used as the control action on the plant until the network recoveries and a new control action packet is received. Equation (9) has involved the compensation of the total transmission delay $r_{k}$ since it compensates the controller-to-actuator transmission delay $r_{k}^{c a}$ in the actuator node and the sensor-to-controller transmission delay $r_{k}^{s c}$ in the controller node when predicting the current state.

\section{STABILITY ANALYSIS}

Define $e_{k \mid k-i}=X_{k}-\hat{X}_{k \mid k-i}$ as the state error between the real state of the plant and the state estimation at instant $k$ generated based the measured state of the plant at instant $k-i$. The method of the stability analysis used here is first to establish an extension model for the current state $X(k)$ and the predicted state errors $e(k)$, obtained by using the latest measured states at instant $k$ with various time delays $r_{k} \in \wp=\left\{\begin{array}{ll}0, & 1,\end{array}, \quad m\right\}$, and then to find a stability condition for the extended model. Therefore, if the extended model is stable under this stability condition the predicted state errors $e(k)$ will approach to zero and the compensated networked control system will be stable under this condition.

The extended state vector, denoted as $Z(k)$, is given in Equation (10).

$$
Z(k)=\left[\begin{array}{c}
X(k) \\
e_{k \mid k-m} \\
e_{k \mid k-m+1} \\
\vdots \\
e_{k \mid k-1}
\end{array}\right]
$$

Because of the random time delay $r_{k} \in \wp=\{0,1, \cdots, m\}$ the latest available plant state used by the controller node for the control action calculation and saved at the actuator node at instant $k$ will be one of $X(k), \quad X(k-1), \cdots$, and $X(k-m)$, depending on the actual total transmission delay $r_{k}$. Equations (11) and (12) give the control actions and the estimations of the state at the same instant $k+1$, but are based on the available measured states at instants $k, k-1, \cdots, k-m+1$ respectively. 


$$
\begin{aligned}
& \left\{\begin{array}{l}
U_{k+1 \mid k}=-K \hat{X}_{k+1 \mid k} \\
U_{k+1 \mid k-1}=-K \hat{X}_{k+1 \mid k-1} \\
\vdots \\
U_{k+1 \mid k-m+1}=-K \hat{X}_{k+1 \mid k-m+1}
\end{array}\right. \\
& \left\{\begin{array}{c}
\hat{X}_{k+1 \mid k}=A X(k)+B U(k) \\
\hat{X}_{k+1 \mid k-1}=A \hat{X}_{k \mid k-1}+B U_{k \mid k-1} \\
\vdots \\
\hat{X}_{k+1 \mid k-m+1}=A \hat{X}_{k \mid k-m+1}+B U_{k \mid k-m+1}
\end{array}\right.
\end{aligned}
$$

Combining Equations (1), (9), (11) and (12) the state errors at instant $k+1$ based on the real measured states at instants $k, k-1, \cdots, k-m+1$ can be given as follows:

$$
\left\{\begin{array}{l}
e_{k+1 \mid k}=(\Delta A-\Delta B K) X_{k}+\Delta B K \sum_{i=1}^{m} \delta\left(r_{k}-i\right) e_{k \mid k-i} \\
\vdots \\
e_{k+1 \mid k-m+1}=(\Delta A-\Delta B K) X_{k}+(A-B K) e_{k \mid k-m+1}+(B+\Delta B) K \sum_{i=1}^{m} \delta\left(r_{k}-i\right) e_{k \mid k-i}
\end{array}\right.
$$

Therefore, by using the notation shown in Equation (10) the extended model of the networked control system with the time-delay compensation can be described by

$$
Z(k+1)=\left[\Lambda+\Delta \Lambda+\left(\Gamma\left(r_{k}\right)+\Delta \Gamma\left(r_{k}\right)\right) L\right] Z(k)
$$

where,

$$
\begin{aligned}
& \Lambda=\left[\begin{array}{ccccc}
A & 0 & \cdots & \cdots & 0 \\
0 & 0 & A & 0 & 0 \\
0 & 0 & 0 & \ddots & \vdots \\
\vdots & \vdots & \vdots & 0 & A \\
0 & 0 & 0 & \cdots & 0
\end{array}\right] \\
& \Delta \Lambda=\left[\begin{array}{cccc}
\Delta A & 0 & \cdots & 0 \\
\Delta A & 0 & \cdots & 0 \\
\vdots & \vdots & \vdots & \vdots \\
\Delta A & 0 & \cdots & 0
\end{array}\right] \\
& \Gamma\left(r_{k}\right)=\left[\begin{array}{ccccc}
-B & \delta\left(r_{k}-m\right) B & \delta\left(r_{k}-m+1\right) B & \cdots & \delta\left(r_{k}-1\right) B \\
0 & \delta\left(r_{k}-m\right) B & \delta\left(r_{k}-m+1\right) B-B & \cdots & \delta\left(r_{k}-1\right) B \\
\vdots & \vdots & \vdots & \ddots & \vdots \\
0 & \delta\left(r_{k}-m\right) B & \delta\left(r_{k}-m+1\right) B & \cdots & \delta\left(r_{k}-1\right) B-B \\
0 & 0 & \cdots & \cdots & 0
\end{array}\right]
\end{aligned}
$$




$$
\begin{gathered}
\Delta \Gamma\left(r_{k}\right)=\left[\begin{array}{cccc}
-\Delta B & \delta\left(r_{k}-m\right) \Delta B & \ldots & \delta\left(r_{k}-1\right) \Delta B \\
-\Delta B & \delta\left(r_{k}-m\right) \Delta B & \ldots & \delta\left(r_{k}-1\right) \Delta B \\
\vdots & \vdots & \vdots & \vdots \\
-\Delta B & \delta\left(r_{k}-m\right) \Delta B & \ldots & \delta\left(r_{k}-1\right) \Delta B
\end{array}\right] \\
L=\operatorname{diag}[\overbrace{K, K, \cdots K}^{m+1}]
\end{gathered}
$$

With the assumption shown in Equations (3) and (4), $\Delta \Lambda$ and $\Delta \Gamma\left(\mathrm{r}_{\mathrm{k}}\right)$ can be expressed as:

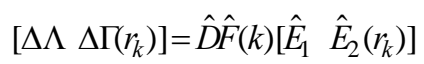

where

$$
\begin{gathered}
\hat{D}=\operatorname{diag}[\overbrace{D, D, \cdots D}^{m+1}] \\
\hat{F}=\operatorname{diag}[\overbrace{F, F, \cdots}^{m+1}] \\
\hat{E}_{1}=\left[\begin{array}{cccc}
E_{1} & 0 & \cdots & 0 \\
E_{1} & 0 & \cdots & 0 \\
\vdots & \vdots & \vdots & \vdots \\
E_{1} & 0 & \cdots & 0
\end{array}\right] \\
\hat{E}_{2}\left(r_{k}\right)=\left[\begin{array}{ccccc}
-E_{2} & \delta\left(r_{k}-m\right) E_{2} & \cdots & \delta\left(r_{k}-1\right) E_{2} \\
-E_{2} & \delta\left(r_{k}-m\right) E_{2} & \cdots & \delta\left(r_{k}-1\right) E_{2} \\
\vdots & \vdots & & \vdots & \vdots \\
-E_{2} & \delta\left(r_{k}-m\right) E_{2} & \cdots & \delta\left(r_{k}-1\right) E_{2}
\end{array}\right]
\end{gathered}
$$

and

$$
\hat{F}^{T}(k) \hat{F}(k) \leq I
$$

The following sufficient stability condition is achieved for the NCS with the predictive compensation shown in Fig. 2. 
Theorem: The NCS shown in Equation (1) with the time-delay compensation described by Equations (7), (8), and (9) is robust stochastically stable if there exist $P_{i}>0, i=1, \cdots, m$, a matrix $L$ described by Equation (15) and a scalar $\varepsilon_{i}>0, i=1, \cdots, m$ satisfying the following $m$ linear matrix inequalities:

$$
M_{i}=\left[\begin{array}{ccc}
-P_{i} & P_{i} L^{T} \Gamma_{i}^{T} W_{i}+P_{i} \Lambda^{T} W_{i} & P_{i} L^{T} \hat{E}_{2}^{T}(i)+P_{i} \hat{E}_{1}^{T} \\
* & -Q+\varepsilon_{i} W_{i}^{T} \hat{D} \hat{D}^{T} W_{i} & 0 \\
* & * & -\varepsilon_{i} I
\end{array}\right]<0
$$

where

$$
\begin{gathered}
W_{i}=\left[\sqrt{P_{i 1}^{r}} I \cdots \sqrt{P_{i m}^{r}} I\right], i=1, \cdots, m . \\
Q=\operatorname{diag}\left\{P_{1}, \cdots P_{m}\right\}
\end{gathered}
$$

$P_{i j}^{r}(j=1, \cdots, m)$ is the corresponding element of the state transition matrix of the Markov process $r_{k}$, as shown in Equation (6). The feedback control matrix $\mathrm{K}$ in Equations (7), (8), and (9) is deterministic and designed without considering the presence of the network in the feedback loop, which assures that Equation (18) is a set of strict linear matrix inequalities and easy to be solved out [16]. The proof of the theorem is given in the appendix.

\section{SIMULATION RESULTS}

Consider a simple discrete plant described in Equations (1), (3) and (4) with the sampling interval $T=0.5$ second and the following parameters

$$
\begin{gathered}
A=\left[\begin{array}{cc}
1.0013 & 0.05 \\
0.05 & 1.0013
\end{array}\right], B=\left[\begin{array}{c}
0.0013 \\
0.05
\end{array}\right] \\
C=\left[\begin{array}{ll}
0.5, & 0.2
\end{array}\right], D=\left[\begin{array}{cc}
0.1 & 0 \\
0 & 0.1
\end{array}\right] \\
E_{1}=\left[\begin{array}{cc}
0.02 & 0.17 \\
0.15 & 0.12
\end{array}\right], E_{2}=\left[\begin{array}{c}
0.1 \\
0.09
\end{array}\right] \\
F(k)=\sin (k) .
\end{gathered}
$$

The network transmission delay $r_{k}$ is a Markov chain with the state space $r_{k} \in \wp=\{0, \quad 1,2,3\}$, and the state transition probabilities matrix is 


$$
P^{r}=\left[\begin{array}{ccc}
0.4 & 0.6 & 0 \\
0.2 & 0.3 & 0.5 \\
0.2 & 0.3 & 0.5
\end{array}\right] .
$$

The total transmission delay $\tau$ is bounded and stochastically varying within $0 \leq \tau \leq m, m=3$. The state feedback controller $U(k)=-K X(k)$, where $K=\left[\begin{array}{ll}10.3 & 3.38\end{array}\right]$, was designed in advance without considering the presence of the network. The controller and the stability condition shown in Equations (7) to (18) can be simplified by setting m=3. For example, Equations (11) and (12) are re-written as

$$
\begin{gathered}
\left\{\begin{array}{c}
U_{k+1 \mid k}=-K \hat{X}_{k+1 \mid k} \\
U_{k+1 \mid k-1}=-K \hat{X}_{k+1 \mid k-1} \\
U_{k+1 \mid k-2}=-K \hat{X}_{k+1 \mid k-2}
\end{array}\right. \\
\left\{\begin{array}{c}
\hat{X}_{k+1 \mid k}=A X(k)+B U(k) \\
\hat{X}_{k+1 \mid k-1}=A \hat{X}_{k \mid k-1}+B U_{k \mid k-1} \\
\hat{X}_{k+1 \mid k-2}=A \hat{X}_{k \mid k-2}+B U_{k \mid k-2}
\end{array}\right.
\end{gathered}
$$

and Equation (10) as

$$
Z(k)=\left[\begin{array}{llll}
X(k) & e_{k \mid k-3} & e_{k \mid k-2} & e_{k \mid k-1}
\end{array}\right]^{T}
$$

and $\hat{E}_{1}$ and $\hat{E}_{2}\left(r_{k}\right)$ as

$$
\begin{gathered}
\hat{E}_{1}=\left[\begin{array}{lll}
E_{1} & 0 & 0 \\
E_{1} & 0 & 0 \\
E_{1} & 0 & 0 \\
E_{1} & 0 & 0
\end{array}\right]_{8 \times 4} \\
\hat{E}_{2}\left(r_{k}\right)=\left[\begin{array}{llll}
-E_{2} & \delta\left(r_{k}-3\right) E_{2} & \delta\left(r_{k}-2\right) E_{2} & \delta\left(r_{k}-1\right) E_{2} \\
-E_{2} & \delta\left(r_{k}-3\right) E_{2} & \delta\left(r_{k}-2\right) E_{2} & \delta\left(r_{k}-1\right) E_{2} \\
-E_{2} & \delta\left(r_{k}-3\right) E_{2} & \delta\left(r_{k}-2\right) E_{2} & \delta\left(r_{k}-1\right) E_{2} \\
-E_{2} & \delta\left(r_{k}-3\right) E_{2} & \delta\left(r_{k}-2\right) E_{2} & \delta\left(r_{k}-1\right) E_{2}
\end{array}\right]_{8 \times 4}
\end{gathered}
$$

Using the LMI toolbox in the MatLab three positive-definite symmetric matrices $P_{1}, P_{2}, P_{3}$ and three scalars $\varepsilon_{1}$, $\varepsilon_{2}, \varepsilon_{3}$ are obtained as follows, which satisfy the linear matrix inequalities shown in Equation (18):

$$
P_{1}=\left[\begin{array}{cccccccc}
0.2243 & -0.5849 & 0.0224 & -0.0062 & 0.0329 & -0.0302 & 0.1055 & -0.0430 \\
-0.5849 & 2.0545 & -0.0419 & -0.0419 & -0.0633 & 0.0190 & -0.0630 & -0.4703 \\
0.0224 & -0.0419 & 538.5407 & -51.1510 & 0.0362 & 0.0101 & 0.0082 & -0.0197 \\
-0.0062 & -0.0419 & -51.1510 & 612.6482 & 0.1304 & -0.4740 & -0.0229 & 0.0552 \\
0.0329 & -0.0633 & 0.0362 & 0.1304 & 319.4949 & 22.2918 & 0.0390 & -0.0462 \\
-0.0302 & 0.0190 & 0.0101 & -0.4740 & 22.2918 & 457.5148 & -0.1675 & 0.3644 \\
0.1055 & -0.0630 & 0.0082 & -0.0229 & 0.0390 & -0.1675 & 8.2486 & -23.3520 \\
-0.0430 & -0.4703 & -0.0197 & 0.0552 & -0.0462 & 0.3644 & -23.3520 & 66.9668
\end{array}\right]
$$




$$
\begin{gathered}
P_{2}=\left[\begin{array}{cccccccc}
0.2622 & -0.6782 & 0.0415 & -0.0157 & 0.0892 & 0.0862 & 0.0776 & 0.0433 \\
-0.6782 & 2.3502 & -0.0900 & -0.0205 & -0.1084 & -0.6874 & -0.1427 & -0.3028 \\
0.0415 & -0.0900 & 564.5135 & -68.8290 & 0.0495 & -0.0490 & 0.0038 & -0.0070 \\
-0.0157 & -0.0205 & -68.8290 & 664.2292 & 0.1238 & -0.4252 & -0.0130 & 0.0253 \\
0.0892 & -0.1084 & 0.0495 & 0.1238 & 26.4723 & -77.1005 & -0.9838 & 2.2817 \\
0.0862 & -0.6874 & -0.0490 & -0.4252 & -77.1005 & 226.2962 & 3.2230 & -7.5407 \\
0.0776 & -0.1427 & 0.0038 & -0.0130 & -0.9838 & 3.2230 & 31.1604 & -75.5287 \\
0.0433 & -0.3028 & -0.0070 & 0.0253 & 2.2817 & -7.5407 & -75.5287 & 186.0335
\end{array}\right] \\
P_{3}=\left[\begin{array}{ccccccccc}
0.2634 & -0.6824 & 0.0248 & 0.2996 & 0.0450 & 0.1865 & 0.0763 & 0.0464 \\
-0.6824 & 2.3970 & -0.0076 & -1.2078 & -0.1016 & -0.6535 & -0.1425 & -0.2743 \\
0.0248 & -0.0076 & 63.3309 & -193.1824 & 0.1226 & -0.1216 & 0.0441 & -0.1682 \\
0.2996 & -1.2078 & -193.1824 & 592.7718 & -0.2390 & -0.1722 & -0.0090 & -0.0039 \\
0.0450 & -0.1016 & 0.1226 & -0.2390 & 56.1316 & -147.1264 & 0.1369 & -0.2738 \\
0.1865 & -0.6535 & -0.1216 & -0.1722 & -147.1264 & 391.3059 & 0.5386 & -1.3909 \\
0.0763 & -0.1425 & 0.0441 & -0.0090 & 0.1369 & 0.5386 & 31.1964 & -75.6111 \\
0.0464 & -0.2743 & -0.1682 & -0.0039 & -0.2738 & -1.3909 & -75.6111 & 186.2531
\end{array}\right]
\end{gathered}
$$

Therefore, according to the stability theorem in Section 4, the NCS with the time-delay compensation is stable for the state feedback controller $U(k)=-K X(k)$, where $K=\left[\begin{array}{ll}10.3 & 3.38\end{array}\right]$.

The responses of the states $x_{1}, x_{2}$, and the output $Y$ under the square wave setpoint change are shown in Figs. 3, 4, and 5 respectively. The system was initially at a steady state, i.e. $x_{1}(0)=0 ; x_{2}(0)=0 ; \quad Y(0)=0$. The setpoint shown in Fig. 5 is changed from 0 to 1.0 at instant $k=0$, and then back from 1.0 to 0 at instant $k=100$. In Figs. 3 and 4 the solid and dash lines represent the responses of the two state variables without and with the transmission delay compensation respectively. It is obvious that the responses with the transmission delay compensation are quicker in approaching to the new steady states and have much less overshoot. Fig. 5 illustrates the same conclusion achieved in the output response. The square wave setpoint is shown in Fig. 5 as a reference. The output with the transmission delay compensation has much less overshoot and approaches to the setpoint much quicker than the one without the compensation. The comparison concludes that the transmission delay compensation method introduced in this paper can improve the system performance.

\section{CONCLUSIONS}

This paper discusses the NCS with a stochastic transmission delay and uncertain process parameters. The stochastic transmission delay is assumed to be a Markov chain and be integer times of the sampling interval. The uncertain parameters are assumed to be norm bounded. A binary variable is introduced to represent the control action with a random transmission delay. A state feedback controller is firstly designed without considering the involvement of the network transmission delay. A buffer is then located in the actuator node to 
save the future control actions sent from the controller node. The control action actually applied to the plant at instant $k+1$ is chosen from the buffer in terms of the total transmission delay. The current time stamp is compared with the time stamp received from the sensor node to compute the current time delay. The buffer is designed to compensate the influence of the transmission delay. An LMI-based sufficient condition for the stability of the NCS with the above compensation is derived in this paper. The simulation results also illustrate the potential of the transmission delay compensation method.

There are still a number of problems to be addressed. Firstly, the stability theorem proposed here is only a sufficient condition. A necessary condition is required in order to identify if there exist a set of parameters satisfying the LMI condition. The state feedback controller is used in this paper for the NCS. If the plant states are un-measurable an output feedback controller for the NCS should be investigated.

\section{ACKNOWLEDGEMENT}

The work was supported by the NSFC grants (60274020, and 69974017) and NSF Hebei (602621). The research grant from the Royal Society in the UK (2005/R1) made the collaboration between Loughborough and HUST possible.

\section{REFERENCES}

1 Yang, S.H, Chen, X. and Alty, J.L: Design issues and implementation of Internet-based process control systems. Control Engineering Practice, 2003, 11, (6), pp. 709-720.

2 Yang, S.H., Chen, X., Tan, L., and Yang, L.: 'Time delay and data loss compensation for Internet-based process control systems', Transactions of the Institute of Measurement and Control, 2005, 27, (2), pp. 103118.

3 Overstreet, J. W. and Tzes, A.: 'An Internet-based real-time control laboratory', IEEE Control Systems Magazine, 1999, 19, (5), pp.320-336.

4 Oboe, R. and Fiorini, P.: 'A design and control environment for internet-based Telerobotics', The International Journal of Robotics Research, 1998, 17, (4), pp. 433-499.

5 Lian, F.L.: 'Analysis, design, modelling and control of networked control systems', PhD dissertation, University of Michigan, USA, 2001.

6 Zhang, W., Branicky, M.S. and Phillips, S.M.: 'Stability of networked control systems', IEEE Control Systems Magazine, 2001, 1, pp. 84-99. 
7 Walsh, G. C., Ye, H., and Bushnell, L.G.: 'Stability analysis of networked control systems', IEEE Transaction on Control System Technology, 2002, 10, (3), pp. 438-446.

8 Montestruque, L. A. and Antsaklis, P. J.: ‘On the model-based control of networked systems', Automatica, 2003, 39, pp. 1837-1843.

9 Luck, R. and Ray, A.: ‘An observer-based compensation for distributed delays’, Automatica, 1990, 26, (5), pp. 903-908.

10 Xiao, G., Yu, S., and Yu, L., MPC-based computer network control strategy with stochastic time delay, IEEE International Conference on SMC, 2003, pp. 3864-3869.

11 Srinivasagupta, D., Schattler, H., and Joseph, B., 'Time-stamped model predictive control: an algorithm for control of processes with random delays', Computers \& Chemical Engineering, 2004, 28, (8), pp. 13371346.

12 Liu, G. P., Rees, D., Chai, S. C., and Nie, X. Y., 'Design, simulation and implementation of networked predictive control systems', Measurement and Control, 2005, 38, (1), pp. 17-21.

13 Wang, Z., Liu, Y., Yang, F., and Liu, X, 'On designing robust controllers under randomly varying sensor delay with variance constraints’, International Journal of General Systems, 2006, 35, (1), pp. 1-15.

14 Wang, Z., Yang, F., Ho, D. W. C. and Liu, X., ‘ Robust $H_{\infty}$ filtering for stochastic time-delay systems with missing measurements', IEEE Transactions on Signal Processing, 2006, 54, (7), pp. 2579-2587.

15 Nilsson, J.: 'Real time control systems with delays', PhD dissertation, Department of Automatic Control, Lund Institute of Technology, Lund, Sweden, 1998.

16 Liu, M.: 'Global exponential stability analysis for neutral delay-differential systems: an LMI approach', International Journal of Systems Science, 2006, 37, (11), pp.777-783.

17 Xie, L. and Souza, C. E. D., 'Robust control for linear systems with norm bounded time-varying uncertainty', IEEE Transactions on Automatic and Control, 1992, 37, pp. 1188-1191.

18 Cao, Y.Y. and Lam, J.: 'Stochastic stability and $H \infty$ control for discrete-time jump linear systems with time delay’, Journal of the Franklin Institute, 1999, 336, pp. 1263-1281.

\section{APPENDIX}

Lemma (see [17]): Let $Z, D, F(t), E$ be matrices with appropriate dimensions. Suppose $Z$ is symmetric and $F^{T}(t) F(t) \leq I$, then

$$
Z+D F(t) E+E^{T} F^{T}(t) D^{T}<0
$$

if and only if there exists scalar $\varepsilon>0$ satisfying 


$$
Z+\varepsilon D D^{T}+\frac{1}{\varepsilon} E^{T} E<0
$$

\section{Proof of the stability theorem in Section 4:}

Combining Equations (14) and (16) the dynamics of the NCS with the time-delay compensation can be represented as follows:

$$
\left\{\begin{array}{c}
Z(k+1)=\Pi\left(r_{k}\right) Z(k) \\
\Pi\left(r_{k}\right)=\Lambda+\Gamma\left(r_{k}\right) L+\hat{D} \hat{F}(k)\left(\hat{E}_{1}+\hat{E}_{2}\left(r_{k}\right) L\right)
\end{array}\right.
$$

If $r_{k}=i$, the matrices $\Pi\left(r_{k}\right), \Gamma\left(r_{k}\right), \hat{E}_{2}\left(r_{k}\right)$ are denoted as $\Pi_{i}, \Gamma_{i}, \hat{E}_{2}(i)$ respectively.

Take the piecewise quadratic stochastic Lyapunov function:

$$
V(\bullet)=Z_{k}^{T} S\left(r_{k}\right) Z_{k}
$$

Where, $S\left(r_{k}\right)=S_{i}>0$, when $r_{k}=i T$.

Let $\bar{S}_{i}=\sum_{j=1}^{m} P_{i j}^{r} S_{j}, G=\operatorname{diag}\left\{S_{1}, \cdots S_{m}\right\}$, and $W_{i}=\left[\sqrt{P_{i 1}^{r}} I \cdots \sqrt{P_{i m}^{r}} I\right], i=1, \cdots m$, where $P_{i j}^{r}$ is the corresponding element of the state transition matrix of the Markov process $r_{k}$. Thus, we have $\bar{S}_{i}=W_{i} G W_{i}^{T}$.

The mean square stable theory of stochastic systems gives [18]:

$$
E\left\{V_{k+1}\left(Z_{k+1}, r_{k+1}\right) \mid Z_{k}, r_{k}=i T\right\}-V_{k}\left(Z_{k}, r_{k}=i T\right)
$$

$$
=Z_{k}^{T}\left(\Pi_{i}^{T} \bar{S}_{i} \Pi_{i}-S_{i}\right) Z_{k}
$$

$$
=Z_{k}^{T}\left(\Pi_{i}^{T} W_{i} G W_{i}^{T} \Pi_{i}-S_{i}\right) Z_{k}
$$

Obviously, if (A.4) <0, the discrete uncertain system is robust stochastically stable. Using the Schur complement the inequality (A.4) $<0$ can be represented as follows:

$$
\left[\begin{array}{cc}
-S_{i} & \Pi_{i}^{T} W_{i} \\
* & -G^{-1}
\end{array}\right]<0
$$

Note that

$$
\begin{aligned}
& {\left[\begin{array}{cc}
-S_{i} & \Pi_{i}^{T} W_{i} \\
* & -G^{-1}
\end{array}\right]=\left[\begin{array}{cc}
-S_{i} & \left(\Lambda+\Gamma_{i} L\right)^{T} W_{i} \\
* & -G^{-1}
\end{array}\right]+\left[\begin{array}{cc}
0 & \left(\hat{D} \hat{F}(k)\left(\hat{E}_{1}+\hat{E}_{2}(i) L\right)\right)^{T} W_{i} \\
* & 0
\end{array}\right]} \\
& =\left[\begin{array}{cc}
-S_{i} & \left(\Lambda+\Gamma_{i} L\right)^{T} W_{i} \\
* & -G^{-1}
\end{array}\right]+\left[\begin{array}{c}
0 \\
W_{i}^{T} \hat{D}
\end{array}\right] \hat{F}(k)\left[\begin{array}{ll}
\hat{E}_{1}+\hat{E}_{2}(i) L & 0
\end{array}\right]+\left[\begin{array}{ll}
\hat{E}_{1}+\hat{E}_{2}(i) L & 0
\end{array}\right]^{T} \hat{F}^{T}(k)\left[\begin{array}{c}
0 \\
W_{i}^{T} \hat{D}
\end{array}\right]^{T}<0
\end{aligned}
$$

According to the Lemma (A.1) and the assumption (17) the inequality (A.5) is true, if and only if there exits a scalar $\varepsilon_{i}>0$ such that

$$
\left[\begin{array}{cc}
-S_{i} & \left(\Lambda+\Gamma_{i} L\right)^{T} W_{i} \\
* & -G^{-1}
\end{array}\right]+\varepsilon_{i}\left[\begin{array}{c}
0 \\
W_{i}^{T} D
\end{array}\right]\left[\begin{array}{c}
0 \\
W_{i}^{T} D
\end{array}\right]^{T}+\frac{1}{\varepsilon_{i}}\left[\hat{E}_{1}+\hat{E}_{2}(i) L \quad 0\right]^{T}\left[\begin{array}{cc}
\hat{E}_{1}+\hat{E}_{2}(i) L & 0
\end{array}\right]<0
$$


which is equivalent to

$$
\left[\begin{array}{ccc}
-S_{i} & \left(\Lambda+\Gamma_{i} L\right)^{T} W_{i} & \left(\hat{E}_{1}+\hat{E}_{2}(i) L\right)^{T} \\
* & -G^{-1}+\varepsilon_{i} W_{i}^{T} \hat{D} \hat{D}^{T} W_{i} & 0 \\
* & * & -\varepsilon_{i} I
\end{array}\right]<0
$$

Let $P_{i}=S_{i}^{-1}$, and $Q=\operatorname{diag}\left\{P_{1}, \cdots P_{m}\right\}$, thus $Q=G^{-1}$. Pre- and post-multiplying the both sides of the inequality (A.6) by $\operatorname{diag}\left[P_{i}, I, I\right]$ leads to the theorem (18). The theorem is proved. 


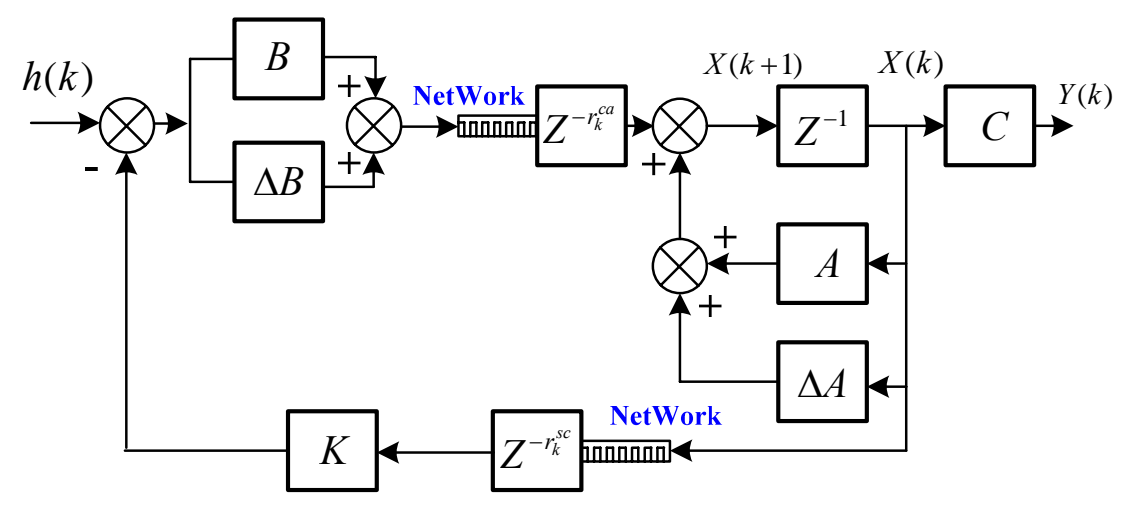

Fig. 1. Networked control system

\section{Buffer}

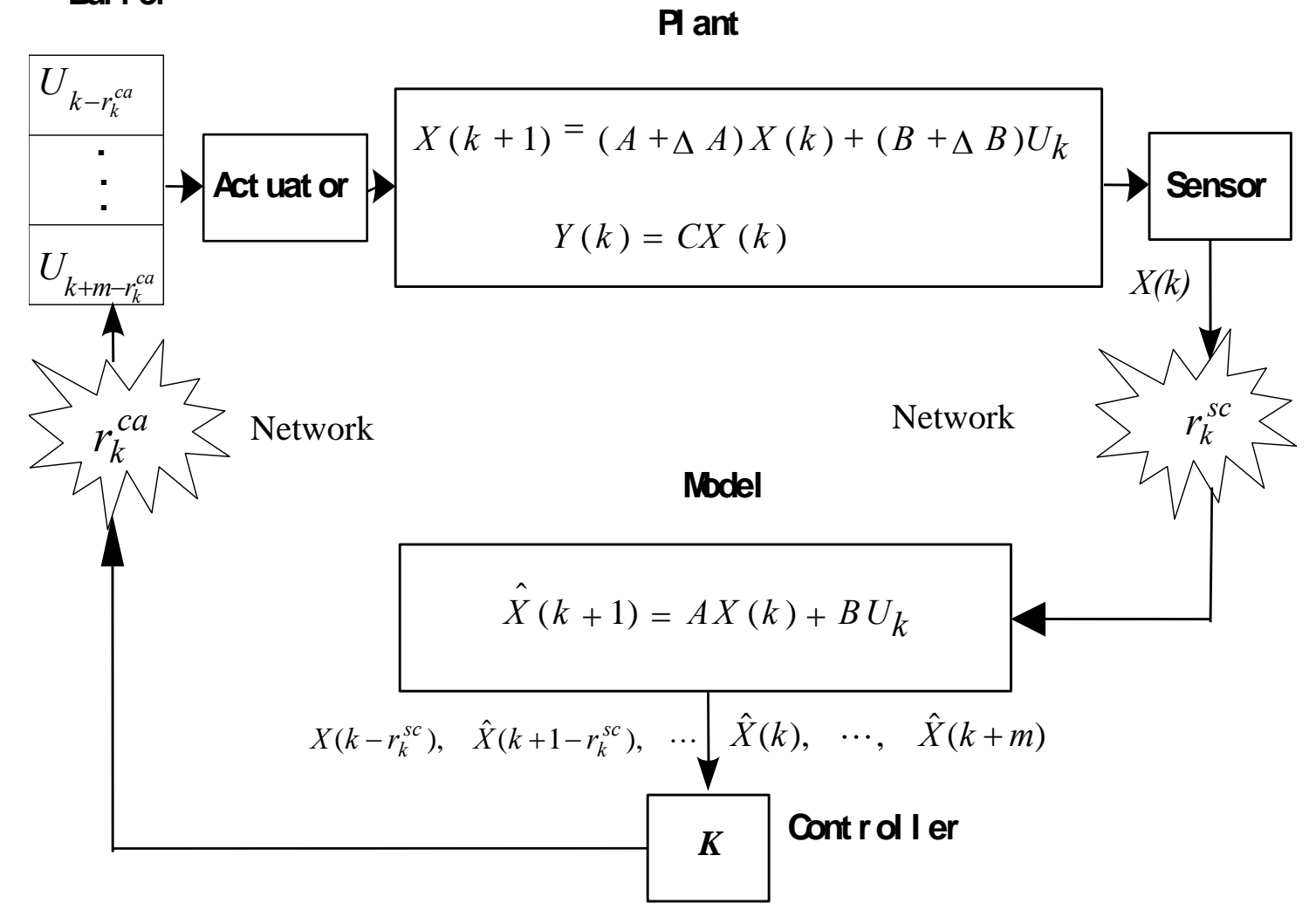

Fig. 2. Transmission delay compensation in the NCS 


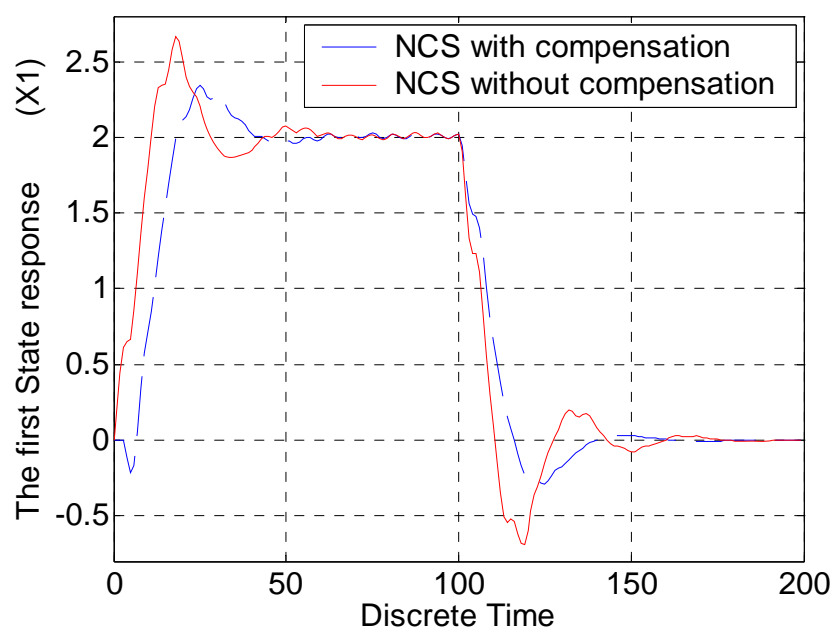

Fig. 3. State $x_{1}$ response

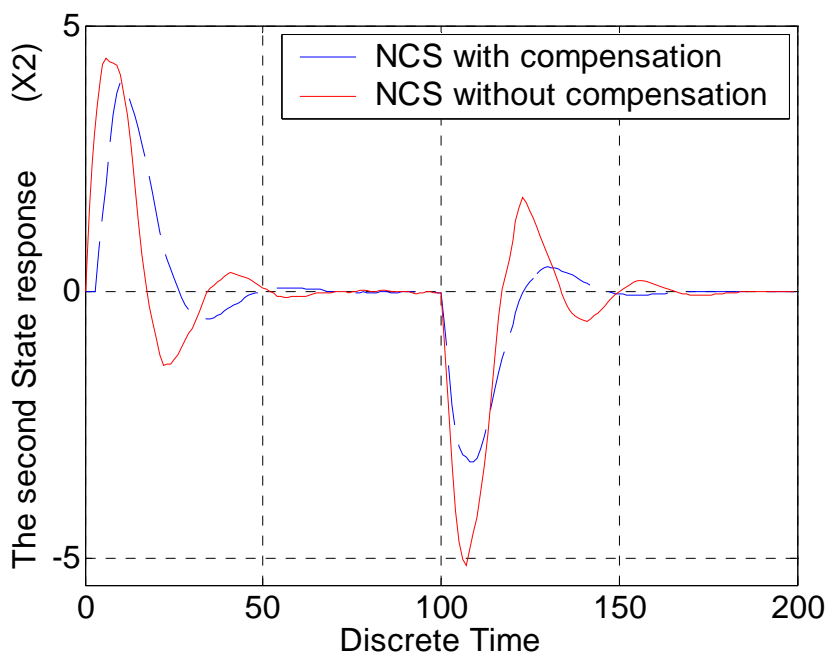

Fig. 4. State $x_{2}$ response

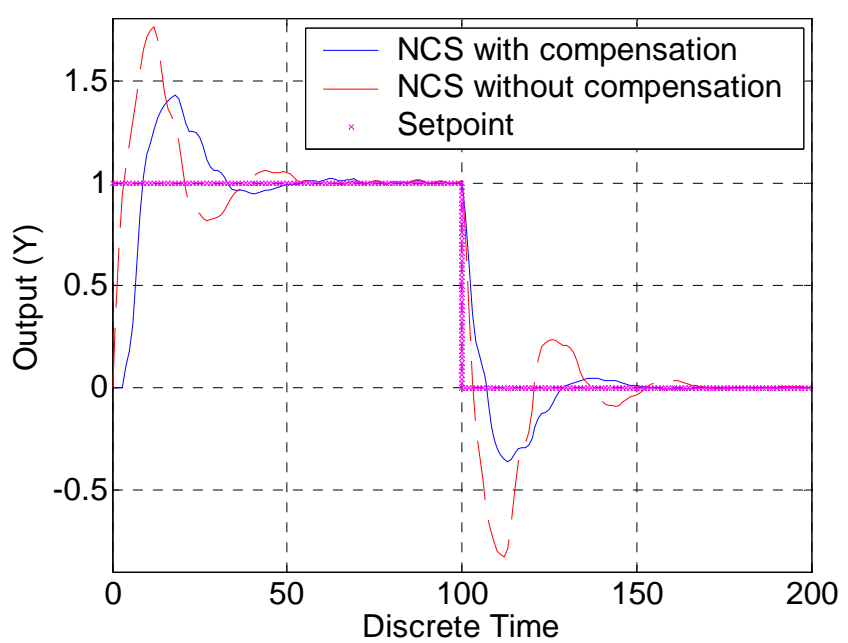

Fig. 5. Output response 